\title{
An underactuated drift-free left-invariant control system on the Lie group ISO $(3,1)$
}

\author{
Camelia Pop ${ }^{1, *}$, and Ioana Iosif $^{1}$ \\ ${ }^{1}$ Politehnica University of Timisoara, Math Department, 2, Victoriei Sq., Romania
}

\begin{abstract}
The purpose of our paper is to study a class of left-invariant, drift-free optimal control problem on the Lie group ISO $(3,1)$. The left-invariant, drift-free optimal control problems involves finding a trajectory-control pair on $I \mathrm{SO}(3,1)$, which minimize a cost function and satisfies the given dynamical constrains and boundary conditions in a fixed time. The problem is lifted to the cotangent bundle $\mathrm{T}^{*} \mathrm{G}$ using the optimal Hamiltonian on $\mathrm{G}^{*}$, where the maximum principle yields the optimal control. We use energy methods (Arnold's method, in this case) to give sufficient conditions for nonlinear stability of the equilibrium states. Around this equilibrium states we might be able to find the periodical orbits using Moser's theorem, as future work. For the some unstable equilibrium states, a quadratic control is considered in order to stabilize the dynamics.
\end{abstract}

\section{Introduction}

The Poincaré group $I S O(3,1)$ was first defined by Minkowski (1908) as the group of space-time isometries. It can be written as a semi-direct product of the Lorentz group $S O(3,1)$ with the four-dimensional translation group $\mathrm{R}^{4}$. Due to its big importance in quantum theory of fields [1-5], we are interested to study an optimal control problem on this Lie group. The interest in such problems arise from their deep applications in engineering (spacecraft dynamics, subaquatic dynamics, the tower control problem), in chemistry (molecular motion control) or physics (quantum theory). The paper's structure is as follows: first, we briefly introduce the Lie algebra $I S O(3,1)$, and its Lie-Poisson structure. In the second paragraph we define an optimal control problem on iso $(3,1)$ and we find the controls that minimize a quadratic cost function. The last paragraphs are dedicated to the stability; first, we analyse the spectral and nonlinear stability of some equilibrium states using energy-Casimir methods; at the last, we use a quadratic cost function to stabilize a family of equilibrium states for which the energy-Casimir methods were inconclusive.

\footnotetext{
* Corresponding author: camelia.ariesanu@upt.ro
} 


\section{The Poincaré Lie algebra iso $(3,1)$}

Let us consider $\left\{J_{i}, K_{i}, P_{i}, H\right\}_{1 \leq i, j \leq 3}$ the usual generators of spatial rotations, boosts, space translations, and time translation respectively, of the Poincaré inhomogeneous Lie algebra iso(3,1) (see [9]); the nonzero brackets are given by

$$
\begin{aligned}
& {\left[J_{i}, J_{j}\right]=\varepsilon_{i j k} J_{k} ;\left[J_{i}, P_{j}\right]=\varepsilon_{i j k} P_{k} ;\left[J_{i}, K_{j}\right]=\varepsilon_{i j k} K_{k} ;} \\
& {\left[H, K_{j}\right]=P_{i} ;\left[K_{i}, K_{j}\right]=-\varepsilon_{i j k} J_{k} ;\left[P_{i}, K_{i}\right]=H .}
\end{aligned}
$$

A general left invariant drift free control system on the Poincaré Lie algebra iso(3,1) with fewer controls than state variables can be written in the following form:

$$
\dot{X}=X\left(\sum_{i=1}^{m} u_{i} A_{i}\right)
$$

where $X \in I S O(3,1)$, the functions $u_{i}$ are the control inputs, $\left\{A_{i}\right\}_{1 \leq i \leq n}$ is the standard basis of the Lie algebra, and $m<10$. In all that follows we shall concentrate to the following leftinvariant, drift-free control system on $i s o(3,1)$ with 4 controls:

$$
\dot{X}=X\left(u_{1} J_{1}+u_{2} J_{2}+u_{3} K_{1}+u_{4} P_{1}\right)
$$

Proposition 1. The system (2) is controllable.

Proof: Since the span of the set of Lie brackets generated by $J_{1}, J_{2}, K_{1}, P_{1}$ coincides with iso $(3,1)$, the Proposition is a consequence of a result due to Jurdjevic and Sussman, see [6].

\section{An optimal control problem for the system (2)}

Let $C$ be the cost function given by:

$$
C\left(u_{1}, u_{2}, u_{3}, u_{4}\right)=\frac{1}{2} \int_{0}^{t_{f}}\left[u_{1}^{2}(t)+u_{2}^{2}(t)+u_{3}^{2}(t)+u_{4}^{2}(t)\right] d t .
$$

The controls that minimize $C$ and steer the system (2) from the initial state $X=X_{0}$ at $t=0$ to the final state $X=X_{f}$ at $t=t_{f}$ are giving by the solutions of the following differential equations:

$$
\left\{\begin{array}{c}
j_{1}^{\prime}=j_{2} j_{3} \\
j_{2}^{\prime}=-j_{1} j_{3}-k_{1} k_{3}-p_{1} p_{3} \\
j_{3}^{\prime}=k_{1} k_{2}+p_{1} p_{2} \\
k_{1}^{\prime}=j_{2} k_{3}+h p_{1} \\
k_{2}^{\prime}=j_{3} k_{1}-j_{1} k_{3} \\
k_{3}^{\prime}=-2 j_{2} k_{1}+j_{1} k_{2} \\
p_{1}^{\prime}=-h k_{1}+j_{2} p_{3} \\
p_{2}^{\prime}=-j_{1} p_{3} \\
p_{3}^{\prime}=j_{1} p_{2}-j_{2} p_{1} \\
h^{\prime}=-k_{1} p_{1}
\end{array}\right.
$$

The system is obtained by applying Krishnaprasad's theorem (see $[6,7,8]$ ) to the optimal Hamiltonian given by: 


$$
H_{o p t}=\frac{1}{2}\left(j_{1}^{2}+j_{2}^{2}+k_{1}^{2}+p_{1}^{2}\right) .
$$

Proposition 2 The dynamics (3) has the following Poisson realization:

$$
\text { (iso } \left.(3,1), \Pi_{-}, H\right) \text {, }
$$

where:

$$
\Pi_{-}=\left(\begin{array}{cccccccccc}
0 & j_{3} & -j_{2} & 0 & k_{3} & -k_{2} & 0 & p_{3} & -p_{2} & 0 \\
-j_{3} & 0 & j_{1} & -k_{3} & 0 & k_{1} & -p_{3} & 0 & p_{1} & 0 \\
j_{2} & -j_{1} & 0 & k_{2} & -k_{1} & 0 & p_{2} & -p_{1} & 0 & 0 \\
0 & k_{3} & -k_{2} & 0 & -j_{3} & j_{2} & h & 0 & 0 & p_{1} \\
-k_{3} & 0 & k_{1} & j_{3} & 0 & -j_{1} & 0 & h & 0 & p_{2} \\
j_{2} & -k_{1} & 0 & -j_{2} & j_{1} & 0 & 0 & 0 & h & p_{3} \\
0 & p_{3} & -p_{2} & -h & 0 & 0 & 0 & 0 & 0 & 0 \\
-p_{3} & 0 & p_{1} & 0 & -h & 0 & 0 & 0 & 0 & 0 \\
p_{2} & -p_{1} & 0 & 0 & 0 & -h & 0 & 0 & 0 & 0 \\
0 & 0 & 0 & -p_{1} & -p_{2} & -p_{3} & 0 & 0 & 0 & 0
\end{array}\right)
$$

is the minus-Lie-Poisson structure on iso $(3,1)$, and

$$
H=\frac{1}{2}\left(j_{1}^{2}+j_{2}^{2}+k_{1}^{2}+p_{1}^{2}\right)
$$

is the Hamiltonian function.

Proof: Indeed, it is not hard to see that the dynamics (3) can be written as

$$
\left(j_{1}^{\prime}, j_{2}^{\prime}, j_{3}^{\prime}, k_{1}^{\prime}, k_{2}^{\prime}, k_{3}^{\prime}, p_{1}^{\prime}, p_{2}^{\prime}, p_{3}^{\prime}, h\right)^{t}=\Pi_{-} \cdot \nabla H,
$$

and $\Pi_{-}$is the minus Lie-Poisson structure on iso $(3,1)$.

Remark Following [9], the Lie-Poisson structure $\Pi_{-}$admits two linear independent Casimir operators:

$$
\begin{gathered}
C_{1}=\frac{1}{2}\left(p_{1}^{2}+p_{2}^{2}+p_{3}^{2}-h^{2}\right) \\
C_{2}=\left(-h j_{3}-p_{1} k_{2}+p_{2} k_{1}\right)^{2}+\left(h k_{2}-p_{1} k_{3}+p_{3} k_{1}\right)^{2}+ \\
+\left(-h j_{1}-p_{2} k_{3}+p_{3} k_{2}\right)^{2}-\left(p_{3} j_{3}+p_{1} j_{1}+p_{2} j_{2}\right)^{2}
\end{gathered}
$$

\section{Stability}

The goal of this section is to analyse the spectral stability and the nonlinear stability of the equilibrium states of the dynamics (3):

$$
\begin{gathered}
e_{1}^{M N P Q R S}=(0,0, M, 0, N, P, 0, Q, R, S), \quad e_{2}^{M N P Q R}=(0,0,0,0, M, N, 0, P, Q, R), \\
e_{3}^{M N P Q}=(0,0, M, 0, N, P, Q, 0,0,0), e_{4}^{M N P Q R S}=(0, M, 0,0, N, 0,0, P, 0, Q), \\
e_{5}^{M N P}=(0,0,0,0, M, N, P, 0,0,0), e_{6}^{M N P}=(M, N, 0,0,0,0,0,0,0, P), \\
e_{7}^{M N P}=(0,0,0, M, 0,0,0, N, P, 0), e_{8}^{M N}=(M, 0,0,0,0,0, N, 0,0,0),
\end{gathered}
$$




$$
e_{9}^{M N}=(M, 0,0,0,0,0,0,0,0, N), e_{10}^{M N}=(M, 0,0,0, N, 0,0,0,0,0) .
$$

Regarding the spectral stability we have established the following results:

Proposition 3 (i) The equilibrium states $e_{1}^{M N P Q R S}$ are spectrally stable for any $M, N, P, Q, R, S \in \mathbb{R}^{*}$.

(ii) The equilibrium states $e_{2}^{M N P Q R}$ are spectrally stable for any reals $M, N, P, Q, R$;

(iii) If $P=0$, then the equilibrium states $e_{3}^{M N 0 Q}$ are spectrally stable iff

$$
M^{2}-Q^{2}<0, N \in \mathbb{R}^{*} \text {. }
$$

(v) The equilibrium states $e_{5}^{M N P}$ are spectrally stable iff $P=0$;

(vi) If $P=0$ or $N=0$ then the equilibrium states $e_{6}^{M N P}$ are spectrally stable;

(vii) If $M=0$ then the equilibrium states $e_{7}^{M N P}$ are spectrally stable;

(viii) The equilibrium states $e_{8}^{M N}$ are spectrally stable iff

$$
M>|N| \sqrt{3+2 \sqrt{2}} \text { or } \mathrm{M}<-|N| \sqrt{3+2 \sqrt{2}}, N \in \mathbb{R}^{*} .
$$

(ix) The equilibrium states $e_{9}^{M N}$ are spectrally stable for any $M, N \in \mathbb{R}^{*}$.

(x) The equilibrium states $e_{10}^{M N}$ are spectrally stable iff $N=0$.

Proof: Let $A$ be the matrix of the linear part of our system:

$$
A=\left(\begin{array}{cccccccccc}
0 & \mathrm{j}_{3} & \mathrm{j}_{2} & 0 & 0 & 0 & 0 & 0 & 0 & 0 \\
-\mathrm{j}_{3} & 0 & -\mathrm{j}_{1} & -\mathrm{k}_{3} & 0 & -\mathrm{k}_{1} & -\mathrm{p}_{3} & 0 & -\mathrm{p}_{1} & 0 \\
0 & 0 & 0 & \mathrm{k}_{2} & \mathrm{k}_{1} & 0 & \mathrm{p}_{2} & \mathrm{p}_{1} & 0 & 0 \\
0 & \mathrm{k}_{3} & 0 & 0 & 0 & \mathrm{j}_{2} & h & 0 & 0 & \mathrm{p}_{1} \\
-\mathrm{k}_{3} & 0 & \mathrm{k}_{1} & \mathrm{j}_{3} & 0 & -\mathrm{j}_{1} & 0 & 0 & 0 & 0 \\
\mathrm{k}_{2} & -2 \mathrm{k}_{1} & 0 & -2 \mathrm{j}_{2} & \mathrm{j}_{1} & 0 & 0 & 0 & 0 & 0 \\
0 & \mathrm{p}_{3} & 0 & -h & 0 & 0 & 0 & 0 & \mathrm{j}_{2} & -\mathrm{k}_{1} \\
-\mathrm{p}_{3} & 0 & 0 & 0 & 0 & 0 & 0 & 0 & -\mathrm{j}_{1} & 0 \\
\mathrm{p}_{2} & -\mathrm{p}_{1} & 0 & 0 & 0 & 0 & -\mathrm{j}_{2} & \mathrm{j}_{1} & 0 & 0 \\
0 & 0 & 0 & -\mathrm{p}_{1} & 0 & 0 & -\mathrm{k}_{1} & 0 & 0 & 0
\end{array}\right)
$$

The corresponding eigenvalues of the linearized $A\left(e_{1}\right)$ are $\lambda_{i}=0, i=\overline{1,6}$ and

$$
\lambda_{7,8,9,10}= \pm \frac{\sqrt{-M^{2}-R^{2}-P^{2}-S^{2}-\sqrt{-4 m^{2} S^{2}+\left(M^{2}+R^{2}+P^{2}+S^{2}\right)^{2}}}}{\sqrt{2}}
$$

so the assertion follows immediately. Similar arguments provides us all the statements.

Let us pass now to the nonlinear stability. We have the following results:

Proposition 4 If $M=N=0$ then the equilibrium states $e_{2}^{M N P Q R}$ are nonlinearly stable.

Proof: In order to prove the nonlinear stability of an equilibrium states, we use the Arnold's method [10]. Let $F_{\mu, \vartheta} \in C^{\infty}\left(\mathbb{R}^{10}, \mathbb{R}\right)$ given by: 


$$
\begin{gathered}
F_{\mu, \vartheta}=H+\mu C_{1}+\vartheta C_{2}= \\
=\frac{1}{2}\left(j_{1}^{2}+j_{2}^{2}+k_{1}^{2}+p_{1}^{2}\right)+\frac{\mu}{2}\left(p_{1}^{2}+p_{2}^{2}+p_{3}^{2}-h^{2}\right)+\frac{\vartheta}{2}\left[\left(-h j_{3}-p_{1} k_{2}+p_{2} k_{1}\right)^{2}+\right. \\
\left.+\left(h h_{2}-p_{1} k_{3}+p_{3} k_{1}\right)^{2}+\left(-h j_{1}-p_{2} k_{3}+p_{3} k_{2}\right)^{2}-\left(p_{3} j_{3}+p_{1} j_{1}+p_{2} j_{2}\right)^{2}\right] .
\end{gathered}
$$

Then, the first variation of $F_{\mu, \vartheta}$ at $e_{2}^{00 P Q R}$ vanished if and only if $\mu=0$ :

$$
\nabla F_{\mu, \vartheta}\left(e_{2}^{00 P Q R}\right)=0 \leftrightarrow \mu=0 .
$$

Considering the space $X=\operatorname{Ker}\left(d C_{1}\right)\left(e_{2}^{00 P Q R}\right) \cap \operatorname{Ker}\left(d C_{2}\right)\left(e_{2}^{00 P Q R}\right), \quad$ then $v^{t} \nabla F_{0, \vartheta}\left(e_{2}^{00 P Q R}\right) v$ is positively for any vector $v=(a, b, c, d, e, f, g, 0,0,0)^{t} \in X$, so $\left.\nabla^{2} F_{\mu, \vartheta}\left(e_{2}^{00 P Q R}\right)\right|_{X \times X}$ is positive defined, therefore $e_{2}^{00 P Q R}$ are nonlinear stable.

Remark: The nonlinear stability of the other equilibrium states remains an open problem, both energy methods being inconclusive.

\section{Stabilization of $e_{8}^{0 N}$ by a quadratic control}

Let us employ the control function $u \in C^{\infty}\left(\mathbb{R}^{10}, \mathbb{R}\right)$,

$$
u=\left(-j_{2} j_{3}, j_{1} j_{3}, 0,-\mathrm{j}_{3} k_{2}, j_{3} k_{1}, 0,-j_{3} p_{2}, j_{3} p_{1}, 0,0\right)
$$

for the system (3). The controlled system (3)-(7), explicitly given by:

$$
\left\{\begin{array} { c } 
{ j _ { 1 } ^ { \prime } = 0 ; } \\
{ j _ { 2 } ^ { \prime } = - k _ { 1 } k _ { 3 } - p _ { 1 } p _ { 3 } } \\
{ j _ { 3 } ^ { \prime } = k _ { 1 } k _ { 2 } + p _ { 1 } p _ { 2 } } \\
{ k _ { 1 } ^ { \prime } = j _ { 2 } k _ { 3 } + h p _ { 1 } } \\
{ k _ { 2 } ^ { \prime } = j _ { 3 } k _ { 1 } - j _ { 1 } k _ { 3 } }
\end{array} \quad \left\{\begin{array}{c}
k_{3}^{\prime}=-2 j_{2} k_{1}+j_{1} k_{2} \\
p_{1}^{\prime}=-h k_{1}+j_{2} p_{3} \\
p_{2}^{\prime}=-j_{1} p_{3} \\
p_{3}^{\prime}=j_{1} p_{2}-j_{2} p_{1} \\
h^{\prime}=-k_{1} p_{1}
\end{array}\right.\right.
$$

has $\mathrm{e}_{8}^{\mathrm{ON}}$ as equilibrium state.

Proposition 5 The dynamics (8) has the following Poisson realization:

$$
\left(\text { iso }(3,1), \Pi_{-}, \widehat{H}\right) \text {, }
$$

where $\Pi_{-}$is the minus Lie-Poisson structure (4) and

$$
\widehat{H}=\frac{1}{2}\left(j_{1}^{2}+j_{2}^{2}+j_{3}^{2}+k_{1}^{2}+p_{1}^{2}\right) .
$$

Proposition 6 The controlled system (8) may be nonlinear stabilized about the equilibrium states $\mathrm{e}_{8}^{0 \mathrm{~N}}$, for any $N \in \mathbb{R}^{*}$.

Proof: In order to prove the nonlinear stability of an equilibrium states, we use the Arnold's method [10]. Let $F_{\mu, \vartheta} \in C^{\infty}\left(\mathbb{R}^{10}, \mathbb{R}\right)$ given by:

$$
\widehat{F}_{\mu, \vartheta}=C_{2}+\vartheta \widehat{H}+\mu C_{1}=\left(-h j_{3}-p_{1} k_{2}+p_{2} k_{1}\right)^{2}+
$$




$$
\begin{gathered}
+\left(h h_{2}-p_{1} k_{3}+p_{3} k_{1}\right)^{2}+\left(-h j_{1}-p_{2} k_{3}+p_{3} k_{2}\right)^{2}-\left(p_{3} j_{3}+p_{1} j_{1}+p_{2} j_{2}\right)^{2} \\
\left.+\frac{\mu}{2}\left(p_{1}^{2}+p_{2}^{2}+p_{3}^{2}-h^{2}\right)+\frac{\vartheta}{2}\left(j_{1}^{2}+j_{2}^{2}+k_{1}^{2}+p_{1}^{2}\right)\right]
\end{gathered}
$$

Then, the first variation of $\widehat{F}_{\mu, \vartheta}$ at $\mathrm{e}_{8}^{0 \mathrm{~N}}$ vanished if and only if $\mu+\vartheta=0$ :

$$
\nabla \widehat{F}_{\mu, \vartheta}\left(\mathrm{e}_{8}^{0 \mathrm{~N}}\right)=0 \leftrightarrow \mu+\vartheta=0 .
$$

Considering the space $X=\operatorname{Ker}\left(d C_{1}\right)\left(\mathrm{e}_{8}^{0 \mathrm{~N}}\right) \cap \operatorname{Ker}(d \widehat{H})\left(\mathrm{e}_{8}^{0 \mathrm{~N}}\right)$, then $\boldsymbol{v}^{t} \nabla \widehat{F}_{\mu, \vartheta}\left(\mathrm{e}_{8}^{0 \mathrm{~N}}\right) \boldsymbol{v}$ is positively for any vector $v=(a, b, c, d, e, f, 0, g, h, i)^{t} \in X$, so $\left.\nabla^{2} \widehat{F}_{\mu, \vartheta}\left(\mathrm{e}_{8}^{0 \mathrm{~N}}\right)\right|_{X \times X}$ is positive defined, therefore $\mathrm{e}_{8}^{0 \mathrm{~N}}$ are nonlinear stable.

\section{Conclusions}

The paper presents a left invariant controllable system on the Lie group $\operatorname{ISO}(3,1)$. Similar problems have been studied for important Lie groups: $S E(2, R) \times S O(2), S E(1,1), S O(4)$, $S E(3, R)$, for a specific Lie group $G_{4}$, and so on. For all this examples, the Poisson geometry approach gives the geometric frame for the study and provide specific methods to obtain stability results, numerical integration using Poisson or non-Poisson integrators or the existence of different type of periodic orbits. Stability results, in our paper, were proved using energy-Casimir methods, more exactly Arnold's method. This involves finding the structure's Casimirs, another specific for Poisson geometry.

\section{References}

1. S. Weinberg, The Quantum Theory of Fields. Vol. 1: Foundations (Cambridge University Press, 1995)

2. M. Maggiore, A Modern Introduction to Quantum Field Theory (Oxford University Press, 2005)

3. W.-K. Tung, Group Theory in Physics (World Scientific, 1985)

4. F. Scheck, Quantum Physics (Springer, 2007)

5. B. Thaller, The Dirac Equation (Springer, 1992)

6. V. Jurdjevic, Optimal control, geometry and mechanics. Mathematical control theory (Springer, New-York, 1999)

7. P.S. Krishnaprasad, Optimal control and Poisson reduction, Technical Report 93-87, Institute for System Research, University of Maryland (1993)

8. A. A. Agrachev, Y. L. Sachkov, Control theory from the geometric viewpoint, Encyclopedia of Mathematical Science, 87. Control Theory and Optimization (SpringerVerlag, 2004)

9. R. Campoamor-Stursberg, A new matrix method for the Casimir operators of the Lie algebras wsp (N, R) and Isp (2N, R), J. Phys. 38(19):4187, DOI 10.1088/03054470/38/19/009 (2005)

10. V. Arnold, Conditions for nonlinear stability of stationary plane curvilinear flows of an ideal fluid, Doklady, 162, 5 (1965). 